Contents lists available at www.ijpba.in

International Journal of Pharmaceutical and Biological Science Archive

PubMed (National Library of Medicine ID: 101738825)

Index Copernicus Value 2017: 71.80

Volume 7 Issue 3; May-June; 2019; Page No. 82-86

\title{
ANALYTICAL METHODS FOR ESTIMATION OF MYCOPHENOLIC ACID IN BULK AND IN PHARMACEUTICAL DOSAGE FORM: A REVIEW
}

\section{Mr. A.U. Manjare* and Mr. Kale R.N.}

Department of Quality Assurance, SVPM's College Of Pharmacy, Malegaon (Maharashtra) India.

Conflicts of Interest: Nil

Corresponding author: Mr. A.U. Manjare

\section{ABSTRACT}

Mycophenolic acid is an anti-metabolite immunosuppressant. It also inhibits the enzyme inosine monophosphate dehydrogenase; essential for purine synthesis. High performance liquid chromatography (HPLC) and the UV are an essential analytical tools in assessing drug product. HPLC methods should be able to isolate, detect, and enumerate the various drugs and drug associated degradants that can form on storage, or manufacturing. It should also detect and enumerate any drugs and drug-related impurities that may be introduced during synthesis. Validation is the process of establishing the performance characteristics and limits of a method and identification of the effects which may change these features and to what extent. This article discusses the current and potential uses of the drug mycophenolic acid as well as the plans and the subjects related to designing UV and HPLC method for development and validation.

Keywords: Mycophenolic acid, Immunosuppressant, HPLC, UV, Validation

\section{Introduction:}

Mycophenolic acid is an immunosuppressive drug used for prevention of rejection in solid organ transplantation. It is not only useful in preventing rejection, being even superior to azathioprine, but also seems to cause less adverse effects than other immunosuppressive drugs. ${ }^{[1]}$ It is one of the few drugs, which were discovered more than a century ago and still in active use. The drug is currently used in patients with liver, lung and bone marrow transplantation. ${ }^{[2]}$ Mycophenolic acid has also been used in renal, rheumatological, gastrointestinal, ophthalmological, dermatological and neurological autoimmune diseases. ${ }^{[2]}$ It is a fungal metabolite that was initially discovered by Bartolomeo Gosio in 1893 as an antibiotic against anthrax bacillus, Bacillus anthracis. ${ }^{[.3]}$ MPA was not and is not used as an antibiotic because of its side effects profile and as there is availability of safer antibiotics. But, studies are still continued on its antibiotic action. ${ }^{[4]}$

Though mycophenolic acid remained out of clinical use for decades after discovery in 1983, the interest of researchers in the molecule continued. Fortunately, the efforts of researchers were not futile. MPA was approved in 1995 by USFDA for the prevention of rejection in renal transplant patients. MPA also possess antiviral ${ }^{[5]}$ and antifungal activities ${ }^{[6]}$ Studies also reported antitumor, and antipsoriasis activities. ${ }^{[7,8]}$ MPA was therefore found as the broad-spectrum acting drug having antiviral, antifungal, antibacterial, anticancer, and antipsoriasis properties. ${ }^{[9]}$ MPA also has antifibrotic effects. ${ }^{[10]}$

To advance oral bioavailability, MPA is administered as mycophenolate mofetil. ${ }^{[11]}$ An oral dose of mycophenolate is hydrolyzed quickly during first pass metabolism to mycophenolic acid which is further metabolized to two minor metabolites namely acyl glucuronide (AcMPAG) and phenolic glucoside of MPA.MPA is highly bound to plasma proteins, mainly to human serum albumin(97-99 \%). ${ }^{[12]}$

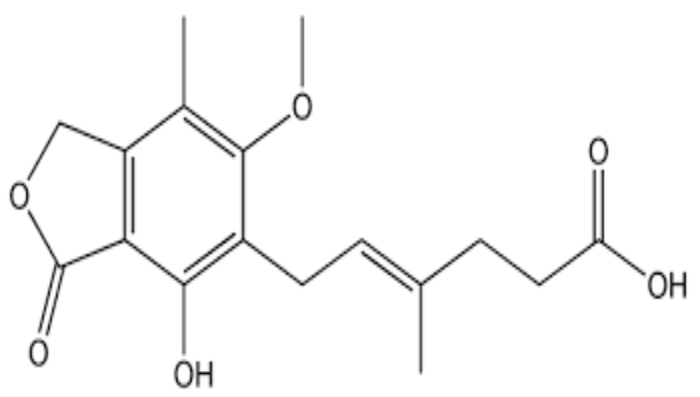

Figure 1: Structure of Mycophenolic Acid

Mycophenolic acid is often used in unification with a calcineurin inhibitor (cyclosporine or tacrolimus) and prednisolone in the primary post-transplant period. ${ }^{[12]}$ MPA hinders inosine monophosphate dehydrogenase, the enzyme that controls the rate 
of synthesis of guanine monophosphate in the de novo trail of purine synthesis used in the proliferation of $B$ and T lymphocytes.

"Chromatography" a overall term for a variety of physicochemical separation techniques all of which have in common the circulation of a component between a mobile phase and a stationary phase.
The method of HPLC flourished after it became possible to create columns with filling materials made of very small beads $(10 \mu \mathrm{m})$ and to utilize them under high pressure. The advance of HPLC and the theoretic understanding of the separation process rest on the basic works of Horvath, Knox, Scott, Snyder, Guiochon, Mockel, and others.

Table 1: Methods for determination of Mycophenolic acid by RP-HPLC and other chromatographic techniques

\begin{tabular}{|c|c|c|c|c|}
\hline Sr. No. & Drug & Method & Description & Reference \\
\hline 1 & $\begin{array}{l}\text { Mycophenolic } \\
\text { acid }\end{array}$ & RP-HPLC method & $\begin{array}{l}\text { Column : USP L7 Octylsilane chemically bonded to porous- } \\
\text { silica } \mathrm{C} 8,(5 \mathrm{~m}),(4.6 \times 250 \mathrm{~mm}) \\
\text { Flow rate: } 1.5 \mathrm{ml} / \mathrm{min} \text {. } \\
\text { Mobile phase : Acetonitrile : Buffer } \quad(50: 50) \\
\text { Buffer: } 0.1 \% \mathrm{v} / \mathrm{v} \text { solution of Orthophosphoric acid. } \\
\text { Diluent : Methanol } \\
\text { Injection volume }: 10 \mathrm{~g} / \mathrm{ml} \\
\text { Detector Wavelength: } 254 \mathrm{~nm} \\
\text { Temperature : } 280 \mathrm{C} \\
\text { Retention time : } 4.872 \mathrm{~min}\end{array}$ & \\
\hline 2 & $\begin{array}{l}\text { Mycophenolic } \\
\text { acid }\end{array}$ & RP-HPLC method & $\begin{array}{l}\text { Column:- } 18 \text { (size- } 250 \times 4.60 \mathrm{~mm} \text {, I.D-5 } \mu \text { ) (Phenomenex) } \\
\text { Flow rate: } 1.2 \mathrm{~mL} / \mathrm{min} . \\
\text { Detector Wavelength: } 216 \mathrm{~nm} \\
\text { Mobile phase : tetra butyl ammonium hydrogen sulphate } \\
\text { and methanol( } 52: 48, \mathrm{v} / \mathrm{v}) \\
\text { Linearity range: } 0.5-160 \mu \mathrm{g} / \mathrm{mL}(\mathrm{r} 2=0.999) \\
\text { LOQ: } 0.321 \mu \mathrm{g} / \mathrm{mL} \\
\text { LOD: } 0.102 \mu \mathrm{g} / \mathrm{Ml}\end{array}$ & 14 \\
\hline 3 & $\begin{array}{l}\text { Mycophenolic } \\
\text { acid }\end{array}$ & $\begin{array}{l}\text { HPLC } \\
\text { assay method }\end{array}$ & $\begin{array}{l}\text { Mobile phase: Acetonitrile: } \\
\text { Sodium acetate buffer }(40: 60 \mathrm{v} / \mathrm{v}) \\
\text { Column:- } \mathrm{C} 18 \text { (size- } 250 \times 4.60 \mathrm{~mm} \text {, I.D-5 } \mu \text { ) (Phenomenex). } \\
\text { Flow Rate:- } 1.0 \mathrm{ml} / \mathrm{min} . \\
\text { Detector :-250nm } \\
\text { Recovery: } 99.86-101.54 \% \\
\text { Injection volume: } 20 \mu \mathrm{L} .\end{array}$ & 15 \\
\hline 4 & $\begin{array}{l}\text { Mycophenolic } \\
\text { acid and } \\
\text { glucuronide } \\
\text { metabolite }\end{array}$ & $\begin{array}{l}\text { its(HPLC-tandem-MS } \\
\text { and an HPLC-UV }\end{array}$ & $\begin{array}{l}\text { Mobile phase: } 20 \mathrm{mmol} / \mathrm{l} \mathrm{NaH} 2 \mathrm{PO} 4 \text { buffer }(\mathrm{pH} 3.0 \text {, adjusted } \\
\text { with } 20 \% \text { phosphoric acid) and methanol }(45: 55, \mathrm{v} / \mathrm{v}) \\
\text { Column:- } \\
\text { Zorbax column }(250 \mathrm{~mm} 4.6 \mathrm{~mm} \text { i.d, } 5 \mathrm{~mm}) \\
\text { Column Temperature- } 45^{\circ} \mathrm{C} \text {, } \\
\text { Flow rate }-1.2 \mathrm{ml} / \mathrm{min} \\
\text { Detector Wavelength: } 304 \mathrm{~nm} \\
\text { Linearity- } 0.2-50 \mathrm{mg} / \mathrm{ml}\end{array}$ & 16 \\
\hline 5 & $\begin{array}{l}\text { Mycophenolic } \\
\text { Acid }\end{array}$ & LC-MS/MS & $\begin{array}{l}\text { Column : Zorbax RP-C18, } 2.1=30 \mathrm{~mm} \\
\text { Linearity - } 30,15 \text { and } 17 \\
\mathrm{Mg} / \mathrm{L} \text {, respectively. } \\
\text { Imprecision }-10 \% \\
\text { flow rate:- } 500 \mathrm{~mL} / \mathrm{min} \\
\text { Mobile Phase:- } 2 \mathrm{mmol} / \mathrm{L} \text { ammonium acetate:water and } \\
\text { methanol }\end{array}$ & 17 \\
\hline 6 & $\begin{array}{l}\text { Mycophenolic } \\
\text { acid }\end{array}$ & HPLC-UV & Mobile phase: $75 \%$ methanol and $25 \%$ ammonium. & 18 \\
\hline
\end{tabular}




\begin{tabular}{|c|c|c|c|c|}
\hline & & & $\begin{array}{l}\text { Column: PFPP column }(50 \mathrm{~mm} \times 2 \mathrm{~mm}, 5 \mathrm{~m}) \text {. } \\
\text { Flow Rate:- } 0.2 \mathrm{ml} / \mathrm{min} . \\
\text { Temperature:-Ambient }\end{array}$ & \\
\hline 7 & \begin{tabular}{|l} 
Mycophenolate \\
Mofetil, \\
Tacrolimus with \\
Prednisolone
\end{tabular} & RP-HPLC Method & $\begin{array}{l}\text { Mobile phase: acetonitrile and } 0.35 \% \text { triethylamine }(\mathrm{pH} \\
\text { 4.2) with Orthophosphoric acid }(70: 30) \\
\text { Column : cKinetex Polar, } \mathrm{C} 18,5 \mu \mathrm{m}, 4.6 \times 250 \mathrm{~mm} \\
\text { Injection volume: } 20 \mu \mathrm{L} . \\
\text { linearity- } 10-100 \mu \mathrm{g} / \mathrm{mL} \\
\text { Flow rate- } 1.2 \mathrm{~mL} / \mathrm{min} . \\
\text { Detector Wavelength: } 254 \mathrm{~nm} \text { for Prednisolone and } \\
\text { Mycophenolate and } 210 \mathrm{~nm} \text { for Tacrolimus. }\end{array}$ & 19 \\
\hline 8 & HPLC-Ms/Ms & HPLC-Ms/Ms & $\begin{array}{l}\text { Column : Phenomenex Kinetex } \mathrm{C} 18(30 \mathrm{~mm} \times 4.6 \mathrm{~mm}, 2.6 \\
\mu \mathrm{m}) \\
\text { Mobile phase: acetonitrile-water } \\
\text { flow rate: } 0.4 \mathrm{~mL} / \mathrm{min} \\
\text { Spray voltage } 3250 \mathrm{~V} \\
\text { Capillary temperature } 222^{\circ} \mathrm{C} \\
\text { Sheath gas } 30 \text { arb. unit } \\
\text { Sweep gas } 2 \text { arb. Unit } \\
\text { Aux Gas } 20 \text { arb. Unit } \\
\text { Vaporizing temperature } 324^{\circ} \mathrm{C} \\
\text { Collision gas pressure } 1.5 \mathrm{~m} \text { Torr } \\
\text { linear range- } 0.5-30 \mu \mathrm{g} / \mathrm{mL} \\
\text { accuracy and precision rang - } 99.76 \text { to } 111.38 \% \\
\text { and from } 2.54 \text { to } 9.01 \%, \text { respectively }\end{array}$ & 20 \\
\hline 9 & $\begin{array}{l}\text { Mycophenolic } \\
\text { Acid }\end{array}$ & $\begin{array}{l}\text { Mycophenolic } \\
\text { Acid }\end{array}$ & $\begin{array}{l}\text { Mobile phase }-54: 46(\mathrm{v} / \mathrm{v}) \text { methanol-0.1\% }(\mathrm{v} / \mathrm{v}) \text { aqueous } \\
\text { trifluoracetic acid. } \\
\text { Flow rate }-1.2 \mathrm{~mL} / \mathrm{min} . \\
\text { Column:- } \\
\text { Kromasil C8 } \\
\text { Column temperature }-40^{\circ} \mathrm{C} \\
\text { Detector Wavelength: } 325 .\end{array}$ & 21 \\
\hline 10 & $\begin{array}{l}\text { Mycophenolic } \\
\text { acid }\end{array}$ & HPLC method & $\begin{array}{l}\text { Mobile phase : } 0.1 \mathrm{M} \text { triethylammonium phosphate } \\
(\mathrm{pH}=5.4) \text {-acetonitrile }(65: 35, \mathrm{v} / \mathrm{v}) \\
\text { Column : } \mathrm{C} 8 \text { analytical }(250 \mathrm{~mm} 4.6 \mathrm{~mm} \text {, particle size } 5 \mu \mathrm{m} \text {; } \\
\text { Perfectsill, } \mathrm{MZ} \text {-Analysen technik, Germany) } \\
\text { Flow rate: } 1.5 \mathrm{ml} / \mathrm{min} . \\
\text { Wavelength-304 } \mathrm{nm} \\
\text { LOD- } 0.05 \mu \mathrm{g} / \mathrm{ml} \\
\text { LOQ- } 0.2 \mu \mathrm{g} / \mathrm{ml} \\
\text { Concentration Range- } 0.2-10 \mu \mathrm{g} / \mathrm{ml}\end{array}$ & 22 \\
\hline 11 & $\begin{array}{l}\text { Mycophenolic } \\
\text { Acid }\end{array}$ & HPLC & $\begin{array}{l}\text { Mobile phase: } 450 \mathrm{Ml} \text { acetonitrile } / 550 \mathrm{~mL} 20 \mathrm{mmol} / \mathrm{L} \\
\text { phosphate buffer, } \mathrm{pH} \\
4.5 \text { ) } \\
\text { Flow rate:- } 1.2 \mathrm{~mL} / \mathrm{min} . \\
\text { Run time:- } 13 \mathrm{~min} \\
\text { retention time }-5.7 \mathrm{~min}\end{array}$ & 23 \\
\hline 12 & $\begin{array}{l}\text { Mycophenolate } \\
\text { mofetil }\end{array}$ & HPTLC method & $\begin{array}{l}\text { Mobile phase- toluene, acetone, and methanol } \\
\text { 6:2:2(V/V/V) } \\
\text { Detector Wavelength:- } 254 \mathrm{~nm} \text {. } \\
\text { Correlation coefficient }-0.9998 \pm 0.0102 \\
\text { LOD }-20.33 \mu \mathrm{g} / \mathrm{ml} \\
\text { LOQ }-60.72 \mu \mathrm{g} / \mathrm{ml}\end{array}$ & 24 \\
\hline
\end{tabular}




\begin{tabular}{|c|c|c|c|c|}
\hline 13 & $\begin{array}{l}\text { Mycophenolate } \\
\text { Mofetil Capsule }\end{array}$ & RP-HPLC METHOD & $\begin{array}{l}\text { Column- Hypersil BDS C18 }(150 \mathrm{~mm} \times 4.6 \mathrm{~mm}, 5 \mu \mathrm{m}) \\
\text { Mobile phase- buffer and Acetonitrile }(650: 350) \mathrm{V} / \mathrm{V} \\
\text { Diluent- Mobile phase } \\
\text { Flow Rate- } 2.00 \mathrm{~mL} / \mathrm{min} \text { Column Temperature- } 50^{\circ} \mathrm{C} \\
\text { Sample temperature- } 20^{\circ} \mathrm{C} \text { Injection Volume- } 10 \mu \mathrm{L} \text { Wave } \\
\text { Length- } 250 \mathrm{~nm} \\
\text { Run time- } 15 \mathrm{~min} \\
\text { USP Plate Count- } 10348.12 \\
\text { Resolution- } 1.658551\end{array}$ & 25 \\
\hline
\end{tabular}

\section{References}

1. Sollinger HW. Mycophenolates in transplantation. Clin Transpl 2004; 18:485-492.

2. Patricia MS, Cees GMK, Coen AS. Use of mycophenolic acid in non- transplant renal diseases. Nephrol Dial transplant 2007;22(4): 1013-1019.

3. Bentley R. Mycophenolic acid: a one hundred year odyssey from antibiotic to immunosuppressant. Chem Rev 2000; 100: 3801-3826

4. Kavanagh F. Activities of twenty-two antibacterial substances against nine species of bacteria. J.Bacteriol. 1947; 54761-54766.

5. Borroto-Esoda K, Myrick F, Feng J, Jeffrey J, Furman P. In-vitro combination of amdoxovir and inosine monophosphate dehydrogenase inhibitors mycophenolic acid and ribavirin demonstrates potent activity against wild-type and drug-resistant variants of human immunodeficiency virus type 1 . Antimicrob Agents Chemother. 2004; 48:4387-94.

6. Nicoletti $R$, De Stefano $M$, De Stefano $S$, Trincone A, Marziano F. Antagonism against Rhizoctonia solani anf fungitoxic metabolite production by some penicillium isolates mycopathologia. 2004; 158:465-474.

7. Tressler RJ, Garvin LJ, Slate DL. Anti-tumor activity of mycophenolate mofetil against human aand mouse tumors in-vivo. Int J Cancer 1994;57:568-573.

8. Epinette WW,Parker CM, Jones EL, Greist MC. Mycophenolic acid for psoriasis. A review of pharmacology, long term efficacy, and safety. J Am Acad Dermatol 1987;17:962-971.

9. Kichin JES,Pomeranz MK, Pak G, Washenik K, Shupack JL. Rediscovering mycophenolic acid: A review of its mechanism, side effects, and potential uses. J Am Acad Dermatol 1997;37(3):445-449.

10. Eugui EM.Fibrogenesis in chronic allograft rejection: underlying mechanism and pharmacological control. Transplant Proc 2002; 34:2867-2871.

11. Ransom JT. Mechanism of action of mycophenolate mofetil. Ther Drug Monit. 1995;17:681-4

12. Nowak I, Shaw LM. Mycophenolic acid binding to human serum albumin: characterization and relation to pharmacodynamics. Clin Chem. 1995;41(7):1011-7.

13. Gopalakrishnan S, Vadivel E, Krishnaveni $P$, Jeyashree B.A Novel reverse phase -HPLC method development and validation of mycophenolate sodium -An immunesuppressant drug. Res J Pharma, Bio Chem Sci 2010; 1(4):200-207.

14. Angirekula N, Mukthinuthalapati MA, Keta RK. Forced degradation studies: A stability indicating liquid chromatographic method for quantification of mycophenolate mofetil in tablets .J Chem Pharma Sci 2017; 10(2):771777.

15. Mehta HS, Singhvi I, Hasumati R .Forced degradation studies and development and validation of stability-indicating RP-HPLC chromatographic method for mycophenolate mofetil assay and related substances. Int $J$ Pharm Pharma Res 2018; 11(4): 14-24.

16. Chen B ,Zhang W, Yu Z, Cai W. Determination of mycophenolic acid(MPA) and its acyl and phenol glucuronide metabolits simultaneously in human plasma by a simplified HPLC method. Analytical Letters 2007; 40: 2465-2475

17. An-sofie $C D$, Favoreel $N$, Fien VH, Verstraete AG. Performance of the roche total mycophenolic acid assay on the cobas integra 400, cobas 6000 and comparision to LC-Ms/Ms in liver transplant patients. Clin Chem Lab Med 2011; 49(7): 1159-1165.

18. Atcheson B, Taylor PJ, Mudge DW, Johson DW, Pillans PI, Tett SE. Quantification of free mycophenolic acid and its glucoronide metabolite in human plasma by liquidchromatography using mass spectrometric and 
ultraviolet absorbance detection. J Chromatogr B 2004;799: 157-163.

19. Sharma PK, Mishra V, Verma S, Bhatia A. Simultaneous estimation by RP-HPLC method for the immunosuppressant drug combination mycophenolate mofetil, tacrolimus with prednisolone. Pertanika J Sci and Technol 2019; 27(1): 371-385.

20. Khokhlov AL, Yaichkov II, Shitav LN, Dzhurko YA, Shitova AM, Ryska $M$ et al. Accurate method of HPLC- Ms/ Ms determination of mycophenolic acid in human plasma. J Bioequiv Availab 2016; 9(1): 306-311.

21. .Xu L,Jiao Z, Liu F, Qiu X,Ji L and Zhang $M$. Pharmacokinetics Evaluation Of Mycophenolic Acid And Its Glucuronide Metabolite In Chinese Renal Transplant Recipients Receiving Enteric Coated Mycophenolate Sodium And Tacrolimus, Therapeutic Drug Monitoring, 2018,1:1-30.

22. Hossein D, Mehrdad H. Simple and sensitive high-performance liquid chromatography (HPLC) Method with UV detection for mycophenolic acid assay in Chuman plasma. Application to a bioequivalence study. Adv Pharm Bull 2015;5(4): 563-568.

23. Shipkova $M$, niedmann PD, Armstrong VW, Schutz E, Wieland E, Shaw LM et al. Simultneous determination of mycophenolic acid and its glucoronide in human plasma using a simple high-performance liquid chromatography procedure. Clin Chem 1998; 44(7): 1481-1488.

24. Kathirvel S, Rajendra Prasad K, Madhu Babu K, Development And Validation of HPTLC Method For The Determination of Mycophenolate Mofetil In Bulk And Pharmaceutical Formulation, Pharmaceutical Methods,2012;3(2):90-93.

25. Vijaya Kumari M, Anusha devi V, Nureshbabu AV,Patan A, Anjaneyulu V, New stability indicating RP-HPLC method for estimation of mycophenolate mofetil capsule in pharmaceutical dosage form, Int Res J Pharm App Sci,2012;2(5):149-154. 